\title{
Radiomics predicts survival of patients with advanced non-small cell lung cancer undergoing PD-1 blockade using Nivolumab
}

\author{
VALERIO NARDONE ${ }^{1}$, PAOLO TINI ${ }^{2}$, PIERPAOLO PASTINA ${ }^{2}$, CIRINO BOTTA $^{3}$, ALFONSO REGINELLI ${ }^{4}$, \\ SALVATORE FRANCESCO CARBONE $^{5}$, ROCCO GIANNICOLA ${ }^{6}$, GRAZIA CALABRESE $^{7}$, CARMELA TEBALA $^{7}$, \\ CESARE GUIDA $^{1}$, ALDO GIUDICE ${ }^{8}$, VITO BARBIERI ${ }^{3}$, PIERFRANCESCO TASSONE ${ }^{3}$, \\ PIEROSANDRO TAGLIAFERRI ${ }^{3}$, SALVATORE CAPPABIANCA $^{4}$, ROSANNA CAPASSO $^{4}$, AMALIA LUCE $^{4}$, \\ MICHELE CARAGLIA ${ }^{4}$, MARIA ANTONIETTA MAZZEI ${ }^{5}$, LUIGI PIRTOLI ${ }^{2}$ and PIERPAOLO CORREALE ${ }^{6}$ \\ ${ }^{1}$ Unit of Radiation Oncology, Integrated Department of Diagnostic Radiology and Radiotherapy, Ospedale del Mare, \\ I-80147 Naples; ${ }^{2}$ Unit of Radiation Oncology, Oncology Department, University Hospital of Siena, I-53100 Siena; \\ ${ }^{3}$ Integrated Area of Medical Oncology, AOU Mater Domini and Department of Experimental and Clinical Medicine, \\ Magna Graecia University of Catanzaro, I- 88100 Catanzaro; ${ }^{4}$ Department of Precision Medicine, University of \\ Campania 'L. Vanvitelli', I-80138 Naples; ${ }^{5}$ Unit of Medical Imaging, Emergency Department and Diagnostic Services, \\ University Hospital of Siena, I-53100 Siena; ${ }^{6}$ Unit of Medical Oncology, Oncology Department, and ${ }^{7}$ Unit of Radiology, \\ Department of Diagnostic Services, Grand Metropolitan Hospital 'Bianchi Melacrino Morelli' Reggio Calabria I-89124; \\ ${ }^{8}$ Epidemiology Unit, IRCCS Istituto Nazionale Tumori ‘Fondazione G. Pascale’, I-80131 Naples, Italy
}

Received February 27, 2019; Accepted August 13, 2019

DOI: $10.3892 / 01.2019 .11220$

\begin{abstract}
Immune checkpoint blockade is an emerging anticancer strategy, and Nivolumab is a human $\mathrm{mAb}$ to PD-1 that is used in the treatment of a number of different malignancies, including non-small cell lung cancer (NSCLC), kidney cancer, urothelial carcinoma and melanoma. Although the use of Nivolumab prolongs survival in a number of patients, this treatment is hampered by high cost. Therefore, the identification of predictive markers of response to treatment in patients is required. In this context, PD-1/PDL1 blockade antitumor effects occur through the reactivation of a pre-existing immune response, and the efficacy of these effects is strictly associated with the presence of necrosis, hypoxia and inflammation at the tumour sites. It has been indicated that these events can be evaluated by specific assessments using a computed tomography (CT) texture analysis (TA) or radiomics. Therefore, a retrospective study was performed, which aimed to evaluate the potential use of this analysis in the identification of patients with NSCLC who may benefit from Nivolumab treatment. A retrospective analysis was performed of 59 patients with metastatic NSCLC who received Nivolumab treatment
\end{abstract}

Correspondence to: Professor Michele Caraglia, Department of Precision Medicine, University of Campania 'L. Vanvitelli', 7 Via de Crecchio, I-80138 Naples, Italy

E-mail: michele.caraglia@unicampania.it

Key words: non-small cell lung cancer, programmed cell death protein 1 , radiomics, texture analysis, survival, nivolumab, immunology between January 2015 and July 2017 at Siena University Hospital (35 patients, training dataset), Catanzaro University Hospital and Reggio Calabria Grand Metropolitan Hospital, Italy (24 patients, validation dataset). Pre- and post-contrast CT sequences were used to contour the gross tumour volume (GTV) of the target lesions prior to Nivolumab treatment. The impact of variations on contouring was analysed using two delineations, which were performed on each patient, and the TA parameters were tested for reliability using the Intraclass Coefficient Correlation method (ICC). All analyses for the current study were performed using LifeX Software $^{\odot}$. Imaging, clinical and pathological parameters were correlated with progression free survival and overall survival (OS) using Kaplan Meier analysis. An external validation testing was performed for the TA Score using the validation dataset. A total of 59 patients were included in the analysis of the present study. The reliability ICC analysis of 14 TA parameters indicated a highly reproducibility (ICC $>0.70$, single measure) in $12(85 \%)$ pre- contrast and $13(93 \%)$ post-contrast exams. A specific cut-off was detected for each of the following parameters: volume (score $1>36 \mathrm{ml}$ ), histogram entropy (score $1>1.30$ ), compacity (score 1 $<3$ ), gray level co-occurrence matrix (GLCM)-entropy (score $1>1.80$ ), GLCM-Dissimilarity (score $1>5$ ) and GLCM-Correlation (score $1<0.54$ ). The global texture score allowed the classification of two subgroups of Low (Score $0-1 ; 36$ patients; 61\%) and High Risk patients (Score $>1$; 23 patients; 39\%) that respectively, showed a median OS of 26 (mean +/- SD: 18 +/- 1.98 months; 95\% CI 14-21 months) and 5 months (mean +/- SD: $6+/-0.99$ months; $95 \% \mathrm{CI}$ : 4-8 months; $\mathrm{P}=0.002$ ). The current study indicated that TA parameters can identify patients that will benefit from PD-1 blockage by defining the radiological settings that are 
potentially suggestive of an active immune response. These results require further confirmation in prospective trials.

\section{Introduction}

Non-small cell lung cancer (NSCLC) represents the most common malignancy and the leading cause of cancer death $(1,2)$. Systemic therapy with platinum doublets with or without Radiation Therapy is the standard frontline treatment for patients in advanced stage of disease (stage IIIB-IV). Patients with driver mutations/rearrangements require molecular targeted specific drugs against EGFR or EML-ALK (3-5) while first line Pembrolizumab an immune checkpoint inhibitor (ICI) can be offered to patients whose tumors have PD-1 ligand-1 (PDL1) expression $>50 \%$. Recently, the clinical development of ICI mAbs has offered new treatment opportunities for NSCLC patients in different settings. In fact, salvage therapy with mAbs to programmed cell death receptor-1 (PD-1) and PDL1 has improved the survival of many of these patients by rescuing pre-existing tumor-specific cytotoxic-T-cells (CTLs) in the tumor sites (6-12). CTLs are the ultimate immune-surveillance system effectors able to recognize and kill tumour cells. PD-1/PDL1 axis is a terminal inflammatory-mediated immune-checkpoint able to protect tumour target cells by delivering an inhibitory signal to tumour-specific CTLs expressing the PD-1 receptor in the tumour site. Experimental evidence have shown that PD-1/PDL1 blockade with specific $\mathrm{mAbs}$ restores the antitumor activity of these tumour-antigen specific CTLs with consequent therapeutic effects in cancer patients. Nivolumab, in particular is a human immune-globulin to PD-1 approved in the treatment of a number of different malignancies including NSCLC, kidney cancer, urothelial carcinoma, and melanoma. Although its use yields a clear benefit and a prolonged survival in a number of patients, this treatment is hampered by high costs, which makes eagerly needed the identification of predictive markers of response, that so far are still undefined and PDL1 analysis has not a clear role in the second line setting. Additionally, its mechanism of action, so different by the conventional cytotoxic treatments or radio/chemotherapy, makes very difficult the monitoring of the patients with the conventional radiology as well as the prevention of possible side effects. In order to identify possible predictive markers of response, we took in consideration that PD-1/PDL1 blockade antitumor effects occur throughout the reactivation of a pre-existing immune response, whose efficacy is strictly related to the presence of necrosis, hypoxia, and inflammation in the tumour sites. We considered that these biological events could be potentially evaluated by specific imaging assessments with a computed tomography (CT) texture analysis (TA) or radiomics.

Radiomics, in particular, is the extraction of quantitative imaging features from medical images. These quantitative values can be used to develop models for cancer diagnosis, patient prognosis, or relative tumor heterogeneity that can then guide clinical decisions (13-15).

This process can be conceptually similar to the current application of tumor staging or genetic and biomolecular information derived from tumor biopsy specimens for clinical decision-making.
TA is performed by means of a computer quantification of both gray-level intensity, position of pixels, and its use is being investigated in several fields (16-23).

In recent years, numerous studies have examined the potential clinical utility of radiomics features calculated from computed tomography (CT) images of NSCLC, correlated with tumor histology (24-26), staging (27), patient prognosis $(19,28-30)$ and genetic mutations (31-33).

On these bases, we have designed a retrospective study to evaluate the potential use of CT TA to predict the OS of pre-treated advanced NSCLC patients treated with PD-1/PDL1 ICI Nivolumab.

\section{Patients and methods}

Patient series. We performed a retrospective analysis of pretreated advanced NSCLC cancer patients treated with Nivolumab between January 2015 and July 2017 at the Radiation Oncology Unit of Siena University Hospital, at the Medical Oncology and Translational Oncology Units of Catanzaro University Hospital and at Reggio Calabria Grand Metropolitan Hospital in Italy.

In our analysis all patients with a complete setting of diagnostic histological samples and a clinical-radiological pre-treatment staging, including a total body CT scan with and without contrast, and an intact (i.e., not previously treated with surgery or radiotherapy) evaluable target lesion in the lung were included.

Ethics approval. All the patients gave written consent to anonymous use of their examinations for research scope. A study notification was submitted to local ethical committee as established by national laws. The University Hospital of Siena Institutional Review Board at the pilot center authorized the retrospective analysis of the data. All procedures were undertaken in compliance with the ethical statements of the Helsinki Declaration (2008) of the World Medical Association.

Nivolumab therapy. All the patients were staged as IIIB/IV and progressed after systemic therapy with cis-platinum doublets. All the patients received intravenous nivolumab (3 $\mathrm{mg} / \mathrm{kg}$ in $60 \mathrm{~min}$ ) on biweekly bases. Treatment was given until progression, unacceptable toxicity or death.

Computed tomography imaging. All CTs at Siena University Hospital were performed using a 64-detector row CT scanner (Discovery 750 HD; GE Healthcare, Milwaukee, WI, USA).

In all patients, $\mathrm{CT}$ of the chest was performed with a spiral technique in tail-cranial direction (from the bases of the lungs to a plane cutting through the upper thoracic outlet, with the patient lying supine). Enhanced CT scans were obtained in the portal venous phase (delay $65-80 \mathrm{sec}$ ) with an intravenous injection of $2 \mathrm{ml} / \mathrm{kg}$ of non-ionic contrast material after a bolus injection of non-ionic contrast material (Iopamidolo $370 \mathrm{mgI} / \mathrm{ml}$; Iopamiro, Bracco Diagnostics, Italy or Iopromide $370 \mathrm{mgI} / \mathrm{ml}$, Ultravist $370 \mathrm{mgI} / \mathrm{ml}$; Bayer HealthCare Pharmaceuticals, Italy), followed by $30 \mathrm{ml}$ of saline solution using a peristaltic semiautomated power injector (3-4 $\mathrm{ml} / \mathrm{sec}$ flow rate; SIAS 757, Bologna, Italy) with an 18-gauge needle in the antecubital vein. The following technical parameters were 
used: slice thickness $2.5 \mathrm{~mm}$, beam pitch $1.375 / 0.937$, reconstruction interval $0.8 \mathrm{~mm}, 120-140 \mathrm{kVp}$ and $250-500 \mathrm{~mA}$. An automatic current modulation tube was used to minimize radiation exposure. Standard reconstruction algorithm was used. Patients were instructed not to breathe during helical imaging in order to avoid motion artefacts.

Follow-up. A CT-scan was repeated, in order to assess the response to immunotherapy, every 3 months or in any case showing clinical signs suggesting progressive disease (PD). General examinations with recording of toxicity, blood cell counts and chemistry and serum markers levels were evaluated every two weeks.

Feature extraction and TA. The gross tumour volume (GTV) was contoured by a radiation oncologist (VN) and confirmed by three expert radiologists (EA, MAM, VR) on pre-contrast and post-contrast sequences (Fig. 1). The impact of variations on contouring was analysed performing two delineation on each patient, and the TA parameters were evaluated for reliability with Intraclass Coefficient Correlation method (ICC). All the analyses for work have been accomplished with LifeX Software $^{\odot}$ (34). TA parameters were limited to features of gray-level co-occurrence matrix (GLCM), sphericity and indices from the gray-level histogram, to avoid an excessive number of parameters and overfitting of models.

Preselection of variables. The reliability analysis performed with ICC showed that the TA parameters that resulted significantly reproducible (ICC $>0.70$, single measure) were, respectively, 12 out of 14 for the post-contrast CT $(85 \%), 13$ out of $14(93 \%)$ for the pre-contrast CT (Table I).

End-points and statistical analysis. In order to perform a statistical correlation among the reliable TA parameters and outcome, we used the software X-Tile (35) to calculate for each TA parameter a cut-off value that could be significant at survival analysis (Kaplan Meier analysis), on the overall population, after normalization of the parameters for the training and validation population.

For each significant cut-off, we assigned the score 1 for the subgroup with the worse prognosis and 0 for the subgroup with the better prognosis.

We then summed the scores to obtain a global texture score that was finally partitioned into two subgroups. The clinical variables of the two subgroups (sex, age, histology) were compared with Chi-square test, in order to exclude biases due to clinical factors not taken into account for the comparison of the above-mentioned subsets.

To validate our performance model, our cohort of patients were separated into two partitions, with the patients treated in our Unit (35 patients) as the training dataset and the patients treated in other Institutions (24 patients) as the validation set.

A survival analysis of progression free survival (PFS) and overall survival (OS) with Kaplan-Meier method was used in the two subgroups to test the texture score.

PFS was calculated from the date of the patient's beginning of immunotherapy to date of CT examination showing progression of disease, or censored to last follow-up visit. Conversely, OS was calculated from the date of the patient's beginning of immunotherapy to the death of patients or censored to last followup visit.

All the statistical analysis was conducted with SPSS software v.23.0.

\section{Results}

Patients' features. This is a retrospective analysis performed on an unmasked sample of fifty-nine consecutive patients with advanced NSCLC cancer who had progressed standard frontline chemotherapy and had received salvage therapy with Nivolumab, between January 2015 and July 2017. We have enrolled $48(81 \%)$ males and $11(19 \%)$ females with a median age of 69 years (mean +-/ SD=67.4 +/- 9.7 years, range 42-86 years). Thirty-six patients $(61 \%)$ were diagnosed as adenocarcinoma, $18(30.5 \%)$ as squamous cell carcinoma and five as undefined NSCLC histology. Forty-five patients were in stage IV (76\%), whereas 14 patients were in stage IIIB (24\%). The description of parameters in the two datasets is presented in Table II.

Median follow-up was 12 months and, on the overall, these patients presented a median PFS of 9 months (mean +-/ $\mathrm{SD}=11$ months +/- 1.3 months, 95\% CI 8.3-13.7 months), and a median OS of 13 months (mean +-/ SD=14.5 months +/1.6 months, 95\% CI 11.1-17.4 months), with 32 (54\%) patients still alive at the last follow-up examination (Fig. 1).

Median cycles of Nivolumab were 10 for the whole population (mean 14, range 2-52 cycles). In our series, survival analysis did not find any correlation with gender (male: Median OS 13 months, mean $14+/-1.8$ months, $95 \%$ CI 10-18 months, vs. female: Median OS 7 months, mean 10 +/- 2.7 months, 95\% CI 5-15 months, P-value: 0.58), with age (Cox-Regression P-value: 0.44), stage (IIIB: Median OS 15 months, mean $14+/-2.4$ months, 95\% CI 10-19 months, vs. IV: Median OS 13 months, mean 12 +/1.7 months, 95\% CI 9-15 months, P-value: 0.60 ) and with histology (squamous cell: Median OS 15 months, mean 14 +/- 2.7 months, 95\% CI 9-20 months, vs. adenocarcinoma: Median OS 13 months, mean 12 +/- 1.6 months, 95\% CI 9-15 months, P-value 0.88).

Survival analysis and texture score. In order to calculate a reproducible cut-off for the targeted TA parameters in CT images, we used the X-Tile Software as described in the Methods section.

A valid parameter cut-off could be selected in the whole population for: 1) volume (score $1>36 \mathrm{ml}$ ); 2) histogram entropy (score $1>1.30), 3$ ) compacity (score $1<3.4$ ), GLCM-entropy (score $1>1.8$ ), 5) GLCM-dissimilarity (score $1>5.6$ ) GLCM-correlation (score $1<0.5$ ). These cut-off values were applied to both training and validation set.

In our analysis, we have restricted the testing of texture parameters describing morphological, histogram and GLCM features, as these parameters have been successfully used and correlated in lung cancer imaging (26), to avoid the risk of overfitting our model using too many parameters (36).

The global texture score, as described in the Methods section, allowed to classify the patients into two cohorts (low risk: Score 0-1, and high risk: Score $>1$ ).

The clinical parameters of the two cohorts were then tested with Chi-square analysis, that demonstrated no statistical differences of the clinical variables in the two subsets, as follows: Age $(\mathrm{P}=0.282)$, sex $(\mathrm{P}=0.843)$, histology $(\mathrm{P}=0.107)$, stage $(\mathrm{P}=0.834)$ (Table II). 
Table I. Reliability analysis of TA parameters.

\begin{tabular}{lcc} 
TA Parameter & Enhanced CT ICC (single measure) & CT ICC (single measure) \\
\hline Volume (ml) & $0.998^{\mathrm{a}}$ & $0.996^{\mathrm{a}}$ \\
Volume & $0.996^{\mathrm{a}}$ & $0.994^{\mathrm{a}}$ \\
Skewness & 0.519 & $0.829^{\mathrm{a}}$ \\
Kurtosis & 0.386 & 0.616 \\
Entropy & $0.879^{\mathrm{a}}$ & $0.968^{\mathrm{a}}$ \\
Energy & $0.808^{\mathrm{a}}$ & $0.841^{\mathrm{a}}$ \\
Sphericity & $0.985^{\mathrm{a}}$ & $0.888^{\mathrm{a}}$ \\
Compacity & $0.994^{\mathrm{a}}$ & $0.989^{\mathrm{a}}$ \\
GLCM-homogeneity & $0.862^{\mathrm{a}}$ & $0.963^{\mathrm{a}}$ \\
GLCM-energy & $0.787^{\mathrm{a}}$ & $0.917^{\mathrm{a}}$ \\
GLCM-contrast & $0.935^{\mathrm{a}}$ & $0.986^{\mathrm{a}}$ \\
GLCM-correlation & $0.707^{\mathrm{a}}$ & $0.847^{\mathrm{a}}$ \\
GLCM-entropy & $0.863^{\mathrm{a}}$ & $0.973^{\mathrm{a}}$ \\
GLCM-dissimilarity & $0.919^{\mathrm{a}}$ & $0.994^{\mathrm{a}}$
\end{tabular}

aParameters that are reliable (ICC >0.7); ICC, Intraclass Coefficient Correlation; TA, texture analysis; GLCM, gray level co-occurrence matrix .
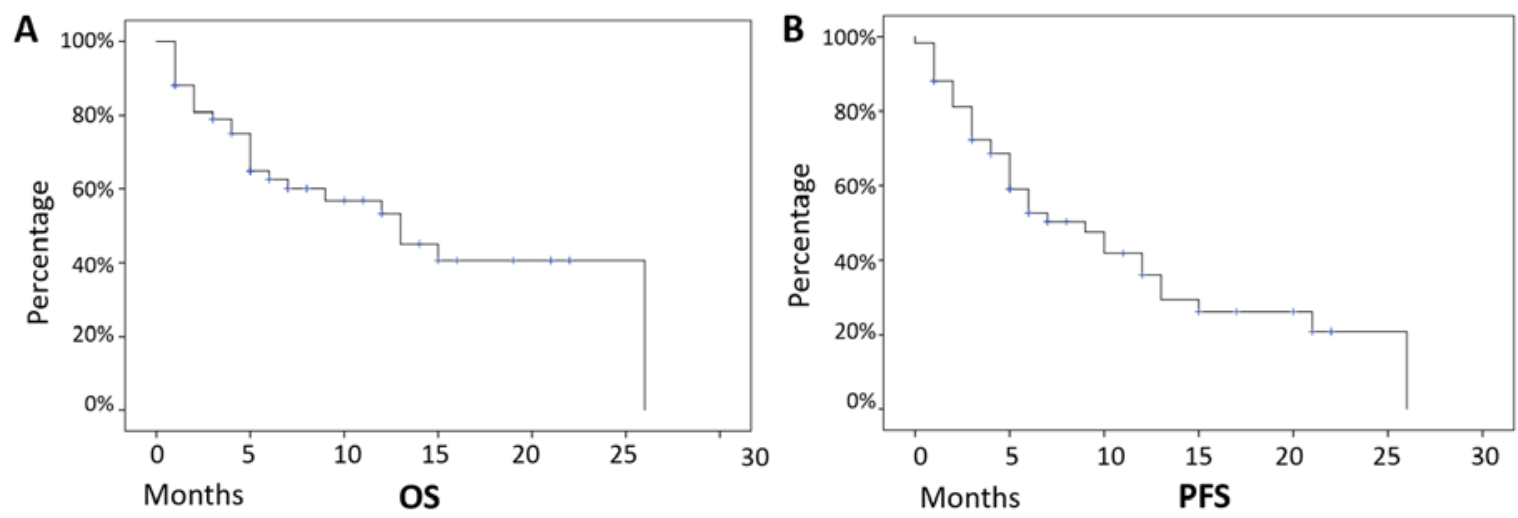

Figure 1. OS and PFS of the whole patient dataset. Median follow-up was equal to 12 months and these patients presented (A) a median PFS of 9 months, and (B) a median OS of 13 months, with 32 (54\%) patients still alive at the last follow-up examination. OS, overall survival; PFS, progression free survival; SD, standard deviation.

PFS resulted significant for both the subsets TRAINING SUBSET (low risk median PFS 10 months, mean $10.9+/-$ 1.8 months, 95\% CI 7-14 months, vs. high risk median PFS 3 months, mean $4.7+/-1.1$ months, $95 \%$ CI 2-6 months, P-value: 0.04 ) and VALIDATION SUBSET (low risk median PFS 15 months, mean $15.5+/-2.9$ months, $95 \%$ CI 9-21 months, vs. high risk median PFS 6 months, mean $6+/-0.9$ months, 95\% CI 4-8 months, P-value: 0.04; Fig. 2).

OS, also, resulted significant in both subsets TRAINING SUBSET (low risk median OS: Not reached, mean 14.4 +/2 months, 95\% CI 10-18 months, vs. high risk median OS: 5 months, mean $6.2+/-1.6$ months, 95\% CI 3-9 months, P-value: 0.02) and VALIDATION SUBSET (low risk median OS: 26 months, mean $19+/-3$ months, 95\% CI 13-25 months, vs. high risk median OS 6 months, mean $6.1+/-0.9$ months, 95\% CI 4-8 months, P-value 0.03; Fig. 2).

The global texture score correlated with patients' survival allowed to classify patients included into two cohorts at low (Score 0-1, 36 patients, 61\%) and high risk (Score> 1,
23 patients, 39\%) of recurrence, respectively, presenting a median PFS of 12 months (mean $13+/-1.7$ months, 95\% CI 9-16 months), vs. a median PFS of 5 months (mean $5.4+/$ 0.7 months, $95 \%$ CI 4-7 months), with a P-value of 0.01, and a median OS of 26 months (mean +/- SD: $17.5+/-1.98$ months, 95\% CI 14-21 months) and 5 months (mean +/- SD: $6+/-$ 0.99 months, $95 \%$ CI: 4-8 months), with a P-value $<0.01$, in the low and high risk groups, respectively (Fig. 2).

Number of cycles of Nivolumab, also, were different among subgroups $(\mathrm{P}=0.026)$, with low risk subset showing a median of 12 cycles (mean 18.5 cycles, range 2-52 cyles) and high risk subset showing a median of 10 cycles (mean 8.8 cycles, range 2-20 cycles).

\section{Discussion}

The recent clinical development of immune-biological drugs such as PD-1/PDL1 inhibitor $m$ Abs for the treatment of common malignancies has clearly risen an extraordinary 
Table II. Patient characteristics.

\begin{tabular}{|c|c|c|c|}
\hline Parameter & Training dataset & Validation dataset & Whole dataset \\
\hline \multicolumn{4}{|l|}{ Sex } \\
\hline Males & $29(83 \%)$ & $19(79 \%)$ & $48(81 \%)$ \\
\hline Females & $6(17 \%)$ & $5(21 \%)$ & $11(19 \%)$ \\
\hline \multicolumn{4}{|l|}{ Age } \\
\hline Median & 71 & 65 & 69 \\
\hline Mean +/- s.d. & $68+/-8$ & $67+/-11$ & $67+/-10$ \\
\hline \multicolumn{4}{|l|}{ Stage } \\
\hline IIIB & $8(23 \%)$ & $6(25 \%)$ & $14(24 \%)$ \\
\hline IV & $27(77 \%)$ & $18(75 \%)$ & $45(76 \%)$ \\
\hline \multicolumn{4}{|l|}{ Histology } \\
\hline Adenocarcinoma & $23(66 \%)$ & $13(54 \%)$ & $36(61 \%)$ \\
\hline Squamous cell & $7(20 \%)$ & $11(46 \%)$ & $18(30.5 \%)$ \\
\hline Not specified & $5(14 \%)$ & & $5(8.5 \%)$ \\
\hline \multicolumn{4}{|l|}{ PFS } \\
\hline Progression & $22(63 \%)$ & $15(62 \%)$ & $37(62 \%)$ \\
\hline No progression & $13(37 \%)$ & $9(38 \%)$ & $22(38 \%)$ \\
\hline \multicolumn{4}{|l|}{ OS } \\
\hline Dead & $15(43 \%)$ & $12(50 \%)$ & $27(45 \%)$ \\
\hline Alive & $20(57 \%)$ & $12(50 \%)$ & $32(55 \%)$ \\
\hline
\end{tabular}

PFS, progression free survival; OS, overall survival.

A
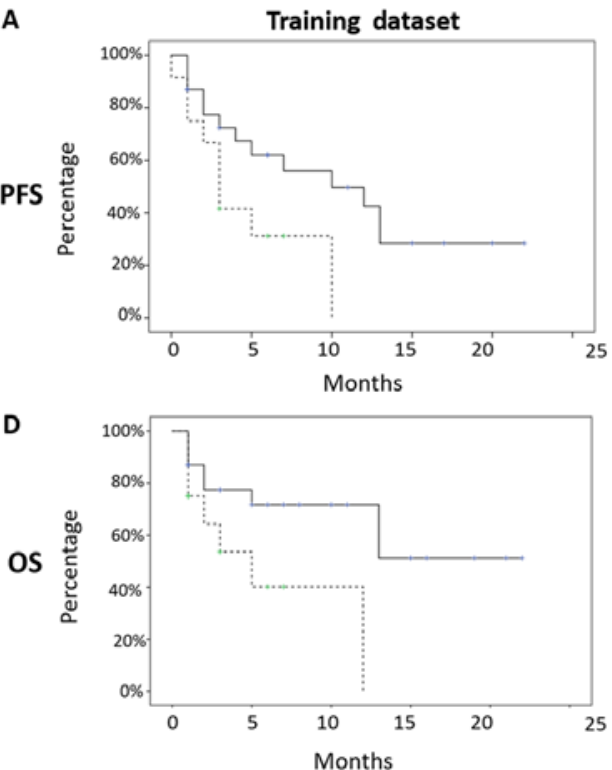

B
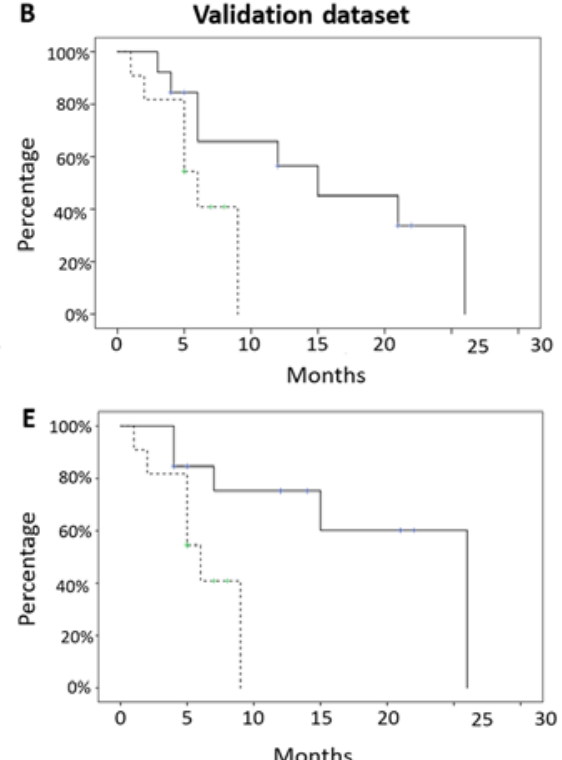
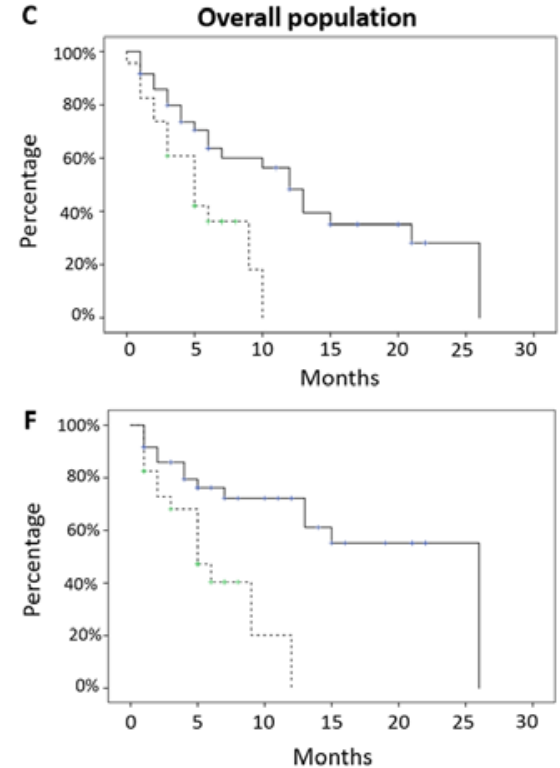

Figure 2. Analysis of survival (OS, PFS) with texture score of the different subsets. PFS was significant for both subsets. (A) TRAINING SUBSET: Low risk PFS: Median PFS 10 months; mean 10.9 +/- 1.8 months; 95\% CI 7-14 months; vs. high risk median PFS 3 months; P=0.044. (B) VALIDATION SUBSET: Low risk median PFS 15 months; vs. high risk median PFS 6 months; $\mathrm{P}=0.048$. OS was significant for both subsets. (D) TRAINING SUBSET: Low risk median OS: Not reached; vs. high risk median OS: 5 months; P=0.023. (E) VALIDATION SUBSET: Low risk median OS: 26 months; vs. high risk median OS 6 months; P-value 0.032. The global texture score, which correlated with patients' survival, allowed the classification of these patients into two cohorts at low (Score 0-1; 36 patients; 61\%) and high risk (Score >1; 23 patients; 39\%). (C): WHOLE COHORT: low risk median PFS of 12 months, vs. high risk median PFS 5 months ( $\mathrm{P}=0.009)$. (F) WHOLE COHORT: low risk median OS 26 vs high risk median OS 5 months $(\mathrm{P}=0.002)$.

challenge in terms of cost, adverse events and patients' monitoring. So far, no clear biomarkers are presently able to select patients who may benefit by treatment with Nivolumab in
NSCLC, although some interesting preliminary results (37). Additionally, these drugs act by turning the immune-balance between tumour and immune system in favour of the latter, 
thus providing a clinical benefit and prolonging patients' survival even in the presence of an apparent radiological progression. This fact makes still more difficult the monitoring of these patients with conventional imaging techniques. In fact, the latter have been designed to evaluate the effects of classical cytotoxic/cytoreductive strategies, as well as chemo-radiotherapy, aimed to provide serial comparisons of tumour volume and number of lesions. In this context, functional imaging techniques, such as diffusion-weighted magnetic resonance imaging (MRI), perfusion methods, positron emission tomography (PET), and radiomics, which are also able to define more qualitative analysis of targeted tumour lesions, are under investigation for providing useful information for these patients $(38,39)$. These techniques are of interest in the search for surrogate biomarkers to potentially define and monitor tumour specific biological processes, including tumour cellularity, growth, necrosis, tumour-associated inflammation and angiogenesis. All these biological events can strictly reflect either tumour progression or responsiveness to the new generation of biological drugs including inhibitors of PD-1/PDL1 immune-checkpoint axis alike Nivolumab, Pembrolizumab, or Atezolizumab mAbs (monoclonal antibodies) (40-44).

In our retrospective multi-institutional analysis, we have restricted the testing of texture parameters to NSCLC patients undergone to salvage treatment with Nivolumab. We have described morphological, histogram and GLCM matrix features defining parameters which have been subsequently correlated in lung cancer imaging, and in recent years with immunotherapy in NSCLC $(26,36,45-49)$. Interestingly, within our sample of NSCLC patients, subjected to Nivolumab treatment, we defined morphological features able to identify a cohort of patients with a very poor prognosis. These patients before the beginning of the immunological treatment had a larger volume of the primary lung lesions associated to a lower compactness, measured as compacity of volume. These combined parameters reflect larger tumour cell burdens paralleled by central hypoxia, necrosis, and invasion beyond the tumour and poor response to cytotoxic treatments. These events additionally define a disease with a very unbalanced antitumour immune response $(19,50,51)$.

In our analysis, a higher histogram entropy was associated with worse outcome. Several reports suggest that this parameter reflects the aggressiveness of the disease as associated to a rapid growth and invasiveness $(27,30,52)$.

The GLCM-entropy, specifically measures the randomness of gray-level voxel pairs, and has been significantly associated, as above, with a worse prognosis in other works $(19,27,30)$.

The GLCM-dissimilarity, on the other hand, measures the variation of gray-level voxel pairs, and can reflect intra-tumour heterogeneity, whereas the GLCM-correlation measures the linear dependence of gray-level in GLCM, and it has been described an important feature in previous studies (19).

These radiomics parameters including higher GLCMentropy and -dissimilarity and lower GLCM-correlations were strictly associated with a worse prognosis. These TA parameters describe a poor immunogenic tumour pattern weakly responsive to PD-1/PDL1 blockade. However, it has to be considered that our results need to undergo critical consideration for many methodological and technical refinements.
In particular, our study is retrospective, and the correlations between textural parameters and clinical outcome requires additional investigations in order to better understand the pathological bases of the TA parameters.

Moreover, the arbitrary choice of the cut-off can lead to significant bias even if we have not unbalanced the subgroups (35) and we have validated our model with an external validation cohort. Conversely, in our series, survival analysis did not show any correlation with gender and age.

In this regard, we believe that the low number of patients analyzed, as well as the low ratio of females (19\%) and the low standard deviation of the age (mean age 67.4 years $+/-9.7$ years) can easily justify these discrepancies with the literature (53). At the same time, the specific subset of patients analyzed (NSCLC patients in progression after first line chemotherapy) could also justify the non-significance of clinical variables such as sex and age.

In conclusion, the predictive value of our TA parameters seems to deserve consideration as a potential and inexpensive biomarker to select NSCLC patients who may benefit by Nivolumab treatment and deserves further development in prospective Randomized trial.

\section{Acknowledgements}

Not applicable.

\section{Funding}

This study was supported by POR CAMPANIA FESR 2014/2020 Sensormircircolar

\section{Availability of data and materials}

The datasets used and/or analyzed during the current study are available from the corresponding author on reasonable request.

\section{Authors' contributions}

All authors have substantially contributed to the conception of the study. VN, PTi, PP, CB, AR, SFC, PC, VB, RG, GC, CT, CG, PTas, PTag, SC, RC, AL, MC, AG, MAM and LP have acquired the data, performed the analysis and interpretation of the data and they have contributed to the drafting and revision of the different versions of the manuscript. All authors have given final approval of the version to be published and agree to be accountable for all the aspects of the work in ensuring that questions related to the accuracy or integrity of any part of the work are appropriately investigated and resolved

\section{Ethics approval and consent to participate}

All patients gave written consent for anonymous use of their examinations for research scope. All procedures were undertaken in compliance with the ethical statements of the Helsinki Declaration (1964, amended most recently in 2008) of the World Medical Association.

\section{Patient consent for publication}

Not applicable. 


\section{Competing interests}

The authors declare that they have no competing interests.

\section{References}

1. Ferlay J, Soerjomataram I, Dikshit R, Eser S, Mathers C, Rebelo M, Parkin DM, Forman D and Bray F: Cancer incidence and mortality worldwide: Sources, methods and major patterns in GLOBOCAN 2012. Int J Cancer 136: E359-E386, 2015.

2. Cetin K, Ettinger DS, Hei YJ and O'Malley CD: Survival by histologic subtype in stage IV nonsmall cell lung cancer based on data from the surveillance, epidemiology and end results program. Clin Epidemiol 3: 139-148, 2011.

3. Pilkington G, Boland A, Brown T, Oyee J, Bagust A and Dickson R: A systematic review of the clinical effectiveness of first-line chemotherapy for adult patients with locally advanced or metastatic non-small cell lung cancer. Thorax 70: 359-367, 2015.

4. Scagliotti GV, Parikh P, von Pawel J, Biesma B, Vansteenkiste J, Manegold C, Serwatowski P, Gatzemeier U, Digumarti R, Zukin M, et al: Phase III study comparing cisplatin plus gemcitabine with cisplatin plus pemetrexed in chemotherapy-naive patients with advanced-stage non-small-cell lung cancer. J Clin Oncol 26: 3543-3551, 2008.

5. Noh JM, Kim JM, Ahn YC, Pyo H, Kim B, Oh D, Ju SG, Kim JS, Shin JS, Hong CS, et al: Effect of radiation therapy techniques on outcome in N3-positive IIIB non-small cell lung cancer treated with concurrent chemoradiotherapy. Cancer Res Treat 48: 106-114, 2016.

6. Luke JJ and Ott PA: PD-1 pathway inhibitors: The next generation of immunotherapy for advanced melanoma. Oncotarget 6 : 3479-3492, 2015.

7. Pardoll DM: The blockade of immune checkpoints in cancer immunotherapy. Nat Rev Cancer 12: 252-264, 2012.

8. Creelan BC: Update on immune checkpoint inhibitors in lung cancer. Cancer Control 21: 80-89, 2014.

9. Horn L, Spigel DR, Vokes EE, Holgado E, Ready N, Steins M Poddubskaya E, Borghaei H, Felip E, Paz-Ares L, et al: Nivolumab versus docetaxel in previously treated patients with advanced non-small-cell lung cancer: Two-year outcomes from two randomized, open-label, phase III trials (CheckMate 017 and CheckMate 057). J Clin Oncol 35: 3924-3933, 2017.

10. Pastina P, Nardone V, Botta C, Croci S, Tini P, Battaglia G, Ricci V, Cusi MG, Gandolfo C, Misso G, et al: Radiotherapy prolongs the survival of advanced non-small-cell lung cancer patients undergone to an immune-modulating treatment with dose-fractioned cisplatin and metronomic etoposide and bevacizumab (mPEBev). Oncotarget 8: 75904-75913, 2017.

11. Pastina P, Nardone V, Croci S, Battaglia G, Vanni F, Bellan C, Barbarino M, Ricci V, Costantini S, Capone F, et al: Anti-cancer activity of dose-fractioned $\mathrm{mPE}+/$ - bevacizumab regimen is paralleled by immune-modulation in advanced squamous NSLC patients. J Thorac Dis 9: 3123-3131, 2017.

12. Nardone V, Pastina P, Giannicola R, Agostino R, Croci S, Tini P, Pirtoli L, Giordano A, Tagliaferri P and Correale P: How to increase the efficacy of immunotherapy in NSCLC and HNSCC: Role of radiation therapy, chemotherapy, and other strategies. Front Immunol 9: 2941, 2018

13. Ganeshan B, Panayiotou E, Burnand K, Dizdarevic S and Miles K: Tumour heterogeneity in non-small cell lung carcinoma assessed by CT texture analysis: A potential marker of survival. Eur Radiol 22: 796-802, 2012

14. Ganeshan B, Abaleke S, Young RC, Chatwin CR and Miles KA: Texture analysis of non-small cell lung cancer on unenhanced computed tomography: Initial evidence for a relationship with tumour glucose metabolism and stage. Cancer Imaging 10: 137-143, 2010.

15. Ganeshan B, Miles KA, Young RC and Chatwin CR: Hepatic enhancement in colorectal cancer: Texture analysis correlates with hepatic hemodynamics and patient survival. Acad Radiol 14 1520-1530, 2007

16. Alobaidli S, McQuaid S, South C, Prakash V, Evans P and Nisbet A: The role of texture analysis in imaging as an outcome predictor and potential tool in radiotherapy treatment planning. Br J Radiol 87: 20140369, 2014.

17. Mattonen SA, Tetar S, Palma DA, Louie AV, Senan S and Ward AD: Imaging texture analysis for automated prediction of lung cancer recurrence after stereotactic radiotherapy. J Med Imaging (Bellingham) 2: 041010, 2015.
18. Mattonen SA, Palma DA, Haasbeek CJ, Senan S and Ward AD: Early prediction of tumor recurrence based on CT texture changes after stereotactic ablative radiotherapy (SABR) for lung cancer. Med Phys 41: 033502, 2014.

19. Coroller TP, Agrawal V, Narayan V, Hou Y, Grossmann P, Lee SW, Mak RH and Aerts HJ: Radiomic phenotype features predict pathological response in non-small cell lung cancer. Radiother Oncol 119: 480-486, 2016.

20. Nardone V, Tini P, Carbone SF, Grassi A, Biondi M, Sebaste L, Carfagno T, Vanzi E, De Otto G, Battaglia G, et al: Bone texture analysis using CT-simulation scans to individuate risk parameters for radiation-induced insufficiency fractures. Osteoporos Int 28: 1915-1923, 2017.

21. Nardone V, Tini P, Nioche C, Biondi M, Sebaste L, Mazzei MA, Banci Buonamici F and Pirtoli L: Texture analysis of parotid gland as a predictive factor of radiation induced xerostomia: A subset analysis. Radiother Oncol 122: 321, 2017.

22. Nardone V, Tini P, Croci S, Carbone SF, Sebaste L, Carfagno T, Battaglia G, Pastina P, Rubino G, Mazzei MA and Pirtoli L: 3D bone texture analysis as a potential predictor of radiation-induced insufficiency fractures. Quant Imaging Med Surg 8: 14-24, 2018.

23. Nardone V, Tini P, Nioche C, Mazzei MA, Carfagno T, Battaglia G, Pastina P, Grassi R, Sebaste L and Pirtoli L: Texture analysis as a predictor of radiation-induced xerostomia in head and neck patients undergoing IMRT. Radiol Med 123: 415-423, 2018.

24. Liu Y, Liu S, Qu F, Li Q, Cheng R and Ye Z: Tumor heterogeneity assessed by texture analysis on contrast-enhanced CT in lung adenocarcinoma: Association with pathologic grade. Oncotarget 8: 53664-53674, 2017.

25. Bae JM, Jeong JY, Lee HY, Sohn I, Kim HS, Son JY, Kwon OJ, Choi JY, Lee KS and Shim YM: Pathologic stratification of operable lung adenocarcinoma using radiomics features extracted from dual energy CT images. Oncotarget 8: 523-535, 2017.

26. Lee G, Lee HY, Park H, Schiebler ML, van Beek EJR, Ohno Y, Seo JB and Leung A: Radiomics and its emerging role in lung cancer research, imaging biomarkers and clinical management: State of the art. Eur J Radiol 86: 297-307, 2017.

27. Craigie M, Squires J and Miles K: Can CT measures of tumour heterogeneity stratify risk for nodal metastasis in patients with non-small cell lung cancer? Clin Radiol 72: 899.e1-899.e7, 2017.

28. Fave X, Zhang L, Yang J, Mackin D, Balter P, Gomez D, Followill D, Jones AK, Stingo F, Liao Z, et al: Delta-radiomics features for the prediction of patient outcomes in non-small cell lung cancer. Sci Rep 7: 588, 2017.

29. Miles KA: How to use CT texture analysis for prognostication of non-small cell lung cancer. Cancer Imaging 16: 10, 2016.

30. Sacconi B, Anzidei M, Leonardi A, Boni F, Saba L, Scipione R, Anile M, Rengo M, Longo F, Bezzi M, et al: Analysis of CT features and quantitative texture analysis in patients with lung adenocarcinoma: A correlation with EGFR mutations and survival rates. Clin Radiol 72: 443-450, 2017.

31. Zhou M, Leung A, Echegaray S, Gentles A, Shrager JB, Jensen KC, Berry GJ, Plevritis SK, Rubin DL, Napel S and Gevaert O: Non-small cell lung cancer radiogenomics map identifies relationships between molecular and imaging phenotypes with prognostic implications. Radiology 286: 307-315, 2018.

32. Liu Y, Kim J, Balagurunathan Y, Li Q, Garcia AL, Stringfield O, Ye Z and Gillies RJ: Radiomic features are associated with EGFR mutation status in lung adenocarcinomas. Clin Lung Cancer 17: 441-448.e6, 2016.

33. Halpenny DF, Plodkowski A, Riely G, Zheng J, Litvak A, Moscowitz C and Ginsberg MS: Radiogenomic evaluation of lung cancer-are there imaging characteristics associated with lung adenocarcinomas harboring BRAF mutations? Clin Imaging 42: 147-151, 2017.

34. Nioche C, Orlhac F, Boughdad S, Reuze S, Soussan M, Robert C, Barakat $\mathrm{C}$ and Buvat I: A freeware for tumor heterogeneity characterization in PET, SPECT, CT, MRI and US to accelerate advances in radiomics. J Nucl Med 58 (Suppl 1): S1316, 2017.

35. Camp RL, Dolled-Filhart $M$ and Rimm DL: X-tile: A new bio-informatics tool for biomarker assessment and outcome-based cut-point optimization. Clin Cancer Res 10: 7252-7259, 2004

36. van der Schaaf A, Xu CJ, van Luijk P, Van't Veld AA, Langendijk JA and Schilstra C: Multivariate modeling of complications with data driven variable selection: Guarding against overfitting and effects of data set size. Radiother Oncol 105: $115-121,2012$. 
37. Giannicola R, D'Arrigo G, Botta C, Agostino R, Del Medico P, Falzea AC, Barbieri V, Staropoli N, Del Giudice T, Pastina P, et al: Early blood rise in auto-antibodies to nuclear and smooth muscle antigens is predictive of prolonged survival and autoimmunity in metastatic-non-small cell lung cancer patients treated with PD-1 immune-check point blockade by nivolumab. Mol Clin Oncol 11: 81-90, 2019.

38. Mazzei MA, Nardone V, Di Giacomo L, Bagnacci G, Gentili F, Tini P, Marrelli D and Volterrani L: The role of delta radiomics in gastric cancer. Quant Imaging Med Surg 8: 719-721, 2018.

39. Nardone V, Reginelli A, Scala F, Carbone SF, Mazzei MA, Sebaste L, Carfagno T, Battaglia G, Pastina P, Correale P, et al: Magnetic-resonance-imaging texture analysis predicts early progression in rectal cancer patients undergoing neoadjuvant chemoradiation. Gastroenterol Res Pract 2019: 8505798, 2019.

40. Lee HY, Jeong JY, Lee KS, Yi CA, Kim BT, Kang H, Kwon OJ, Shim YM and Han J: Histopathology of lung adenocarcinoma based on new IASLC/ATS/ERS classification: Prognostic stratification with functional and metabolic imaging biomarkers. J Magn Reson Imaging 38: 905-913, 2013.

41. Kim YN, Lee HY, Lee KS, Seo JB, Chung MJ, Ahn MJ, Park K, Kim TS and Yi CA: Dual-energy CT in patients treated with anti-angiogenic agents for non-small cell lung cancer: New method of monitoring tumor response? Korean J Radiol 13: 702-710, 2012.

42. van Elmpt W, Zegers CML, Reymen B, Even AJG, Dingemans AC, Oellers M, Wildberger JE, Mottaghy FM, Das M, Troost EGC and Lambin P: Multiparametric imaging of patient and tumour heterogeneity in non-small-cell lung cancer: Quantification of tumour hypoxia, metabolism and perfusion. Eur J Nucl Med Mol Imaging 43: 240-248, 2016.

43. Chi JT, Thrall DE, Jiang C, Snyder S, Fels D, Landon C, McCall L, Lan L, Hauck M, MacFall JR, et al: Comparison of genomics and functional imaging from canine sarcomas treated with thermoradiotherapy predicts therapeutic response and identifies combination therapeutics. Clin Cancer Res 17: 2549-2560, 2011.

44. Tini P, Nardone V, Pastina P, Pirtoli L, Correale P and Giordano A: The effects of radiotherapy on the survival of patients with unresectable non-small cell lung cancer. Expert Rev Anticancer Ther 18: 593-602, 2018 .
45. Virginia BM, Laura F, Silvia R, Roberto F, Francesco F, Eva H, Charles F, Samy A, Stefan M, Jean-Charles S, et al: Prognostic value of histogram analysis in advanced non-small cell lung cancer: A radiomic study. Oncotarget 9: 1906-1914, 2018.

46. Bera K, Velcheti V and Madabhushi A: Novel quantitative imaging for predicting response to therapy: Techniques and clinical applications. Am Soc Clin Oncol Educ Book 38: 1008-1018, 2018.

47. Trebeschi S, Drago SG, Birkbak NJ, Kurilova I, Cǎlin AM, Pizzi AD, LalezariF, Lambregts DMJ, Rohaan M, Parmar C, et al: Predicting response to cancer immunotherapy using non-invasive radiomic biomarkers. Ann Oncol, Mar 21, 2019 (Epub ahead of print).

48. Harmon S, Seder CW, Chen S, Traynor A, Jeraj R and Blasberg JD: Quantitative FDG PET/CT may help risk-stratify early-stage non-small cell lung cancer patients at risk for recurrence following anatomic resection. J Thorac Dis 11: 1106-1116, 2019.

49. Shi L, He Y, Yuan Z, Benedict S, Valicenti R, Qiu J and Rong Y: Radiomics for response and outcome assessment for non-small cell lung cancer. Technol Cancer Res Treat 17: $1533033818782788,2018$.

50. Einenkel J, Braumann UD, Horn LC, Pannicke N, Kuska JP, Schütz A, Hentschel B and Höckel M: Evaluation of the invasion front pattern of squamous cell cervical carcinoma by measuring classical and discrete compactness. Comput Med Imaging Graph 31: 428-435, 2007.

51. Nardone V, Nanni S, Pastina P, Vinciguerra C, Cerase A, Correale $P$, Guida C, Giordano A, Tini P, Reginelli A, et al: Role of perilesional edema and tumor volume in the prognosis of non-small cell lung cancer (NSCLC) undergoing radiosurgery (SRS) for brain metastases. Strahlenther Onkol 195: 734-744, 2019.

52. Grove O, Berglund AE, Schabath MB, Aerts HJ, Dekker A, Wang H, Velazquez ER, Lambin P, Gu Y, Balagurunathan Y, et al: Quantitative computed tomographic descriptors associate tumor shape complexity and intratumor heterogeneity with prognosis in lung adenocarcinoma. PLoS One 10: e0118261, 2015.

53. Liao Y, Fan X and Wang X: Effects of different metastasis patterns, surgery and other factors on the prognosis of patients with stage IV non-small cell lung cancer: A surveillance, epidemiology, and end results (SEER) linked database analysis. Oncol Lett 18: 581-592, 2019. 